\title{
EVALUACIÓN DE SILOS CONTINUOS DE HOMOGENIZACIÓN EN LA INDUSTRIA DEL CEMENTO, UTILIZANDO TÉCNICAS DE RADIOTRAZADORES
}

\author{
Carlos Sebastián Calvo \\ carlos.sebastianc@urp.pe \\ Universidad Ricardo Palma
}

\begin{abstract}
RESUMEN
Se ha estudiado el proceso de homogenización continua, en un silo de alta capacidad de procesamiento, con el objetivo de evaluar los parámetros que determinan su eficiencia en operación continua. El método permite alcanzar la operación óptima a través de la observación del movimiento y distribución de diferentes fracciones de crudo fino marcadas con La-140 como trazador. Se discute el cambio de homogeneidad en función del tiempo y también de influencia del diseño del silo en el grado de homogenización. La experiencia se desarrolla utilizando un trazador preparado con la materia prima cruda y fina, mezclada uniformemente con $40 \mathrm{mCi}$ de La-140 en la forma química de $\mathrm{La}_{2} \mathrm{O}_{3^{\prime}}$ e introducida al silo mediante un pulso dirigido hacia la zona de carga. Se realizaron mediciones de la concentración del trazador a la salida del silo. Se demuestra de la influencia de la operación del silo en la homogenización y en la provisión de cemento de buena calidad y dentro de las especificaciones técnicas exigidas.
\end{abstract}

PalABRAS ClaVE: Homogenización, radiotrazador, detector, adquisición de datos, distribución del tiempo de residencia, desviación estándar, grado de homogenización.

\section{EVALUATION OF CONTINUOUS SILOS OF BLENDING IN THE CEMENT INDUSTRY USING RADIOTRACER TECHNIQUES}

\begin{abstract}
Pneumatic blending process in a large capacity blending silo has been studied, in order to evaluate the parameters connected with its efficiency in continuous operation. The method allows obtaining optimal operation through the observation of movement and distribution of the different crude fractions labelled with Lanthanum 140 as a radiotracer. Changes in blending factors are discussed as a function of time as well as the influence of silo design in the degree of homogenization. The experiment was carried out by the aid of a tracer prepared with fine crude raw material mixed uniformly with $40 \mathrm{mCi}$ of La 140 in the chemical form of $\mathrm{La}_{2} \mathrm{O}_{3^{\prime}}$ injecting this in a pulse into the charging zone. Concentration measurements were performed at the outlet of the silo. The results showed the influence of the operation of the silo in the blending effect, to provide good quality cement, in good agreement with specific standards.
\end{abstract}

KEYWORDS: Blending, radiotracer, probe, data acquisition, residence time distribution, standard deviation, homogenization degree. 


\section{Fundamentos teóricos}

La distribución del tiempo de residencia o la frecuencia de la distribución de edades para una corriente de material que atraviesa un recipiente son dos nombres diferentes de la misma función $\mathrm{E}(\mathrm{t})$. La función se normaliza de tal forma que:

$$
\int_{0}^{\infty} E(t) d t=1
$$

El tiempo de residencia se obtiene a partir de:

$$
\bar{t}=\int_{0}^{\infty} t \cdot E(t) d t
$$

siendo $\mathrm{E}$ el tiempo medio de residencia.

Así mismo, cuando la señal de entrada es un impulso Dirac, el tiempo medio se puede obtener a partir de la curva de concentración del trazador a la salida del proceso (Curva C), obteniéndose en este caso:

$$
\bar{t}=\frac{\int_{0}^{\infty} t C(t) d t}{\int_{0}^{\infty} C(t) d t}
$$

Se sabe también que el tiempo de residencia promedio geométrico se puede determinar como el cociente de la masa $m$ contenida en el silo y el flujo másico $\dot{m}$. Considerando que la densidad del material permanece prácticamente constante, es una alternativa posible tomar el cociente del volumen lleno $V$ y el flujo volumétrico $Q$ que atraviesa el medio, como se expresa en la Ecuación 4.

$$
\bar{t}_{g}=\frac{m}{\dot{m}}=\frac{V}{Q}
$$

, siendo V/Q el llamado tiempo medio de permanencia o tiempo medio de residencia en el caso de sistemas ideales.

La Ecuación 4 rendirá los mismos resultados que la Ecuación 2 si la masa total m o el volumen total $V$ del silo, participan completamente en el proceso de mezcla. Si solamente una parte del silo participa activamente (volumen efectivo del silo), se obtiene un valor exacto para $\mathrm{E}$ a partir de la Ecuación 2 mientras la Ecuación 4 lo sobre estima. Por lo tanto con la ayuda de mediciones de los tiempos de residencia, será posible también determinar el volumen efectivo del silo.

En el caso de un sistema operando normalmente con todo su volumen utilizado, no habría volúmenes muertos. Sin embargo, algunas regiones del sistema pueden presentar tiempos de tránsito de 5 a 10 veces el tiempo de retención del resto del material, pudién- 
dose considerar como inactivas o muertas para los fines prácticos. El volumen muerto presente en el sistema se estima con la ecuación:

$$
\% V_{\text {muerto }}=\left(1-\frac{\bar{t}_{\text {exp }}}{\bar{t}_{\text {teor }}}\right) 100=\left(1-\frac{V_{\text {efect }}}{V}\right) 100
$$

; donde $V_{\text {efect }} \mathrm{y} V$ son los volúmenes efectivo y geométrico del sistema, $\mathrm{E}_{\text {exp }}$ es el tiempo medio de residencia obtenido experimentalmente a partir de la curva de distribución del tiempo de residencia (DTR), con utilización de las Ecuaciones 2 o 3 y $\mathrm{E}_{\text {teor }}$ es el tiempo medio de residencia geométrico obtenido con la Ecuación 4.

La desviación standard, expresada por la Ecuación 6, fue adoptada del criterio establecido para la no homogeneidad de la mezcla:

$$
\sigma=\sqrt{\frac{\sum_{i=1}^{n}\left(C_{i}-C_{m}\right)^{2}}{n-1}}
$$

Donde $n$ es el número de muestras, $C_{i}$ la concentración de las muestras individuales, $C_{m}$ la media aritmética de la concentración de todas las muestras.

Se ha encontrado una relación exponencial entre la desviación estándar y el tiempo de mezcla, la misma que se indica en la Ecuación 7

$$
\frac{\sigma^{2}}{\sigma_{0}^{2}}=e^{-A t}
$$

donde:

$\sigma=$ Desviación relativa de las concentraciones de la muestra al tiempo «t»

$\sigma_{0}=$ Desviación relativa de las concentraciones de la muestra antes del mezclado $A=$ Constante de proporcionalidad con la intensidad de mezcla $\mathrm{t}=$ tiempo de mezclado

Luego, la homogeneidad de una mezcla es caracterizada por la desviación estándar de la concentración de componentes encontrados en diferentes muestras, mientras que un proceso de mezcla es caracterizado por el cambio de dicha desviación en función del tiempo. De la Ecuación 7 se deduce que el tiempo de mezcla media puede ser calculado de $t=\ln 2 / A$ 


\section{Sistema bajo investigación}

Para una mejor comprensión de la experiencia y sus repercusiones en la optimización del proceso, es preciso conocer de manera genérica, la secuencia de la fabricación; la misma que se resume a seguir.

Las materias primas para la fabricación del cemento, es decir la arcilla, mineral de hierro y calizas, provienen de canteras cercanas a la planta, luego son dosificadas e ingresan a sistemas de reducción de tamaño constituidos por chancadoras y molinos de bolas y posteriormente a un sistema de ciclones para separar los finos. Por la parte inferior de los ciclones se separa el crudo fino o «harina cruda» y por la parte superior los productos muy finos que van a un sistema de electro-filtros donde por alto voltaje se energizan las partículas recuperándose una parte como crudo fino.

El crudo fino es conducido al techo de un enorme silo de homogenización. Por la parte inferior sale el crudo homogenizado, el cual luego de ganar calorías provenientes de los gases de cabeza del horno rotatorio, ingresa al horno rotatorio por cuyo extremo opuesto sale el clinker. Este clinker es enfriado por aire, reducido de tamaño y dosificado con yeso para obtener el cemento.

El silo investigado es usado tanto para el proceso de homogenización continua como para almacenamiento de mezcla fina. La alimentación continua al silo tiene lugar a través de una canaleta principal de distribución y seis canaletas de entrada, ubicados en la parte superior del silo, asegurando una distribución controlada de los diferentes estratos de material (Fig.1).

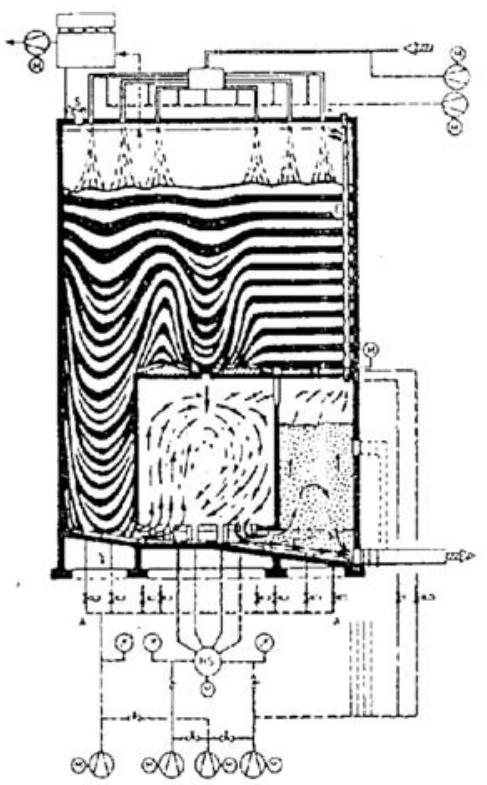

Fig.1 Esquema del modo de funcionamiento del silo de homogenización investigado 
Como se observa, los diferentes estratos con diferente composición química, se desplazan formando embudos hacia las aberturas entrada ubicada en la parte superior de una cámara interna y en la parte inferior de la misma, produciendo un primer efecto de homogenización. Se considera el arreglo interno del silo continuo como si cortáramos estratos de crudo fino de diferente composición química y los colocásemos dentro de un recipiente más pequeño para ser mezclados. De esta manera el material se mezclará lo mismo en la cámara interna, como si la operación se realizara en el silo total.

Como la homogenización tiene lugar en la cámara interna, el aire requerido por $\mathrm{m}^{3}$ de material será aproximadamente el mismo y debido a los relativos pequeños volumen y altura en la cámara interna, el volumen y presión de aire requerido para el proceso de homogenización se reduce enormemente, con el consecuente ahorro de energía; utilizándose compresores de baja potencia para la cámara interna en vez de grandes compresores para el silo completo. Esa es la razón por la cual en el proceso de homogenización continua predomina hoy en día el proceso de mezclado por cuadrantes.

El sistema de aireación por cuadrantes se encuentra ubicado en el piso del silo. Cada cuadrante opera secuencialmente como cuadrante de mezcla mientras los otros tres sirven como cuadrantes de aireación Compresores suministran aire, tanto para aireación como para mezcla. En estas condiciones se crea una columna de material de baja densidad completamente aireada, encima del cuadrante de mezcla. La columna de material más denso sobre los cuadrantes de aireación se combina continuamente con el material de la columna de mezcla, que tiene menor densidad y se desplaza hacia arriba creando una circulación vertical muy activa de material, el cual se desvía hacia un compartimiento adyacente de almacenamiento de material homogenizado, ubicado al costado de la cámara interna. A intervalos regulares de tiempo los cuadrantes se alternan en sus funciones, por lo que el suministro de aire también es cíclico en los cuadrantes.

\section{目 Almacenamiento y descarga \\ $\square \quad$ Sin aireación \\ ¿ Cuadrante activo, con aireación \\ 田 Cuadrantes inactivos, sin aireación}

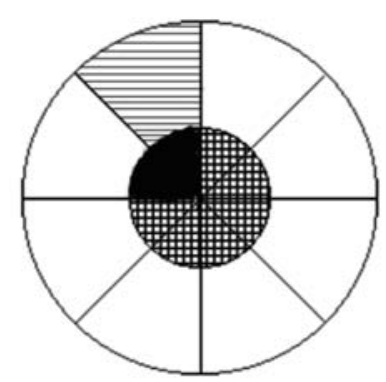

Fig. 2 Funcionamiento del sistema de aireación mediante cuadrantes 
El material así mezclado continuamente fluye hacia la salida del silo por dos aberturas de descarga en el presente caso. La descarga de material es igual a la carga fresca de crudo fino del silo. La Fig.2 muestra la disposición de planta de los cuadrantes del silo, durante el funcionamiento.

\section{Diseño experimental}

El crudo fino disponible tiene las siguientes características:

- Densidad aparente

$0,8117 \mathrm{~kg} / \mathrm{dm}^{3}$ (sin compactar) $1,830 \mathrm{~kg} / \mathrm{dm}^{3}$ (compactada)

- Porcentaje retenido en mallas: (promedios mensuales)

\begin{tabular}{|c|c|c|c|c|c|}
\hline Medición & \multicolumn{5}{|c|}{$\operatorname{Micras}(\mathrm{m})^{*}$} \\
\hline & 212 & 150 & 90 & 63 & 45 \\
\hline 1 & $0,8 \%$ & $3,6 \%$ & $19,6 \%$ & $24,8 \%$ & $39,6 \%$ \\
\hline 2 & $0,8 \%$ & $4,4 \%$ & $19,6 \%$ & $29,2 \%$ & $42,8 \%$ \\
\hline
\end{tabular}

*Optimo: 16 a 18\% de retención en malla de 90 m

- Contenido de humedad: $0,2 \%$

Teóricamente, el crudo fino debe salir del silo con una composición química que depende de la composición química de las materias primas (calizas, arcilla y mineral de fierro) y de la abundancia relativa de las mismas, en la carga. Las materias primas ingresan con las composiciones siguientes:

Composición química (\%)

\begin{tabular}{|l|c|r|r|r|r|r|c|}
\hline Materias primas & $\mathrm{SiO}_{2}$ & $\mathrm{Al}_{2} \mathrm{O}_{3}$ & $\mathrm{Fe}_{2} \mathrm{O}_{3}$ & \multicolumn{1}{c|}{$\mathrm{Ca} 0$} & $\mathrm{MgO}$ & $\mathrm{SO}_{3}$ & P.F. \\
\hline Caliza Alta & 10,02 & 3,37 & 0,82 & 47,02 & 0,35 & 0,68 & 38,70 \\
\hline Caliza Baja & 20,83 & 3,98 & 1,68 & 38,91 & 0,70 & 0,52 & 32,07 \\
\hline Arcilla & 43,64 & 27,55 & 6,50 & 5,00 & 0,40 & 0,43 & 16,22 \\
\hline Mineral de Fierro & 32,20 & 6,55 & 24,20 & 15,15 & 0,65 & 0,37 & 16,78 \\
\hline
\end{tabular}

Las materias primas entran al proceso con la siguiente abundancia relativa:

Caliza alta ( 80 a $88 \%$ de $\left.\mathrm{CaCO}_{3}\right)$ $70 \%$

Caliza baja ( 60 a $80 \%$ de $\mathrm{CaCO}_{3}$ ) $20 \%$

Mineral de Hierro $\left(25 \% \mathrm{Fe}_{2} \mathrm{O}_{3^{\prime}}, 30\right.$ a $40 \% \mathrm{SiO}_{2}$

7 a $8 \% \mathrm{Al}_{2} \mathrm{O}_{3^{\prime}} 20 \% \mathrm{CaCO}_{3^{\prime}} \mathrm{Mn}$, sulfuros, etc.)

Arcilla (30 $\mathrm{Al}_{2} \mathrm{O}_{3}$ y $43 \% \mathrm{SiO}_{2}$ ) 
Con esta información es fácil determinar la composición química que deberá tener la harina cruda homogenizada al salir del silo, obteniéndose los siguientes resultados: $\mathrm{SiO}_{2}=13,78 \% ; \mathrm{Al}_{2} \mathrm{O}_{3}=3,82 \% ; \mathrm{Fe}_{2} \mathrm{O}_{3}=2,05 \% ; \mathrm{CaO} \quad=42,91 \% ; \mathrm{MgO}=0,41 \% ; \mathrm{SO}_{3}=0,64$ $\%$; P.F. $=36,01 \%$.

La muestra trazadora debe tener similares características físicas a las indicadas para el crudo fino. La selección del radioisótopo y la actividad a inyectar así como otras variables referidas al trazador, se determinaron teniendo en cuenta el volumen del silo, los flujos de entrada y salida máximos, la capacidad probable de detección eficaz final y otras consideraciones de seguridad nuclear y protección radiológica.

El equipamiento principal consistió en un analizador de radiación RD-1600 Wallac un terminal de datos, un graficador X-Y y dos detectores de Nal(TI) 2»X2».La capacidad de almacenamiento del silo llenado a un $80 \%$ de su volumen durante la experiencia era de 1800 Ton. Por otro lado el flujo de crudo, tanto en la entrada como en la salida era de 220 Ton/h durante las determinaciones. En estas condiciones se tendría un tiempo de residencia teórico medio de 8,2 h., aplicando la Ecuación 4.

Con estas consideraciones, se definieron las características siguientes para el radiotrazador utilizado:

- Muestra trazadora:

- Isótopo:

- Actividad:

- Forma química:

- Forma física:

- Granulometría del $\mathrm{Fe}_{2} \mathrm{O}_{3}$ :

- Peso de la muestra trazadora:

- Punto de inyección:

- Punto de detección:
Muestra de crudo fino proveniente de los ciclones, con radioisótopo incorporado, La-140, $T_{1 / 2}=40$ horas, $\quad$ Tipo de emisión: $\gamma$ Energía: 1,6 MeV

$40 \mathrm{mCi}$

$\mathrm{La}_{2} \mathrm{O}_{3}$ En polvo

$19,6 \%$ para malla de $90 \mu$ (similar a la harina cruda proveniente de los ciclones)

$10 \mathrm{~kg}$

Muestreador del «Air Lift» (acumulador para elevación del crudo hacia el techo del silo)

En c/u de las ventanas ubicadas en las dos canaletas de salida del crudo del silo (parte inferior)

- Tiempo total estimado de las determinaciones: 2 días en planta.

\section{Procedimiento experimental}

La disposición de la instalación evaluada se muestra en la Fig.4, en donde se indican además sus dimensiones y los puntos de inyección y detección del radiotrazador. El trazador fue inyectado en el sistema de transporte de material, en un punto cercano al elevador por aire; el mismo que desplaza el material rápidamente, conforme se verificó con un 
detector ubicado en la canaleta de alimentación ubicada en el techo del silo. La respuesta de esta detección inicial fue similar a un impulso delta de Dirac, Debido al rápido tránsito del trazador desde el punto de inyección hasta su ingreso al silo, se ha considerado el momento de inyección como el tiempo cero o tiempo de inicio de las mediciones en el silo de homogenización. En la misma Fig.3 se muestra además una vista de tope del techo del silo en donde se aprecia el sistema de carga constituido por una canaleta de alimentación y seis canaletas de distribución que reparten uniformemente la carga al silo.

Por otro lado, existen dos canaletas de salida de producto homogenizado por la parte inferior del silo, las cuales están provistas de ventanas que se pueden abrir sin provocar pérdidas de material. En las ventanas de cada una de las canaletas se colocó un detector de radiación a fin de comparar los resultados obtenidos y verificar posibles influencias de los cambios alternados de los cuadrantes de aireación, descritos anteriormente.

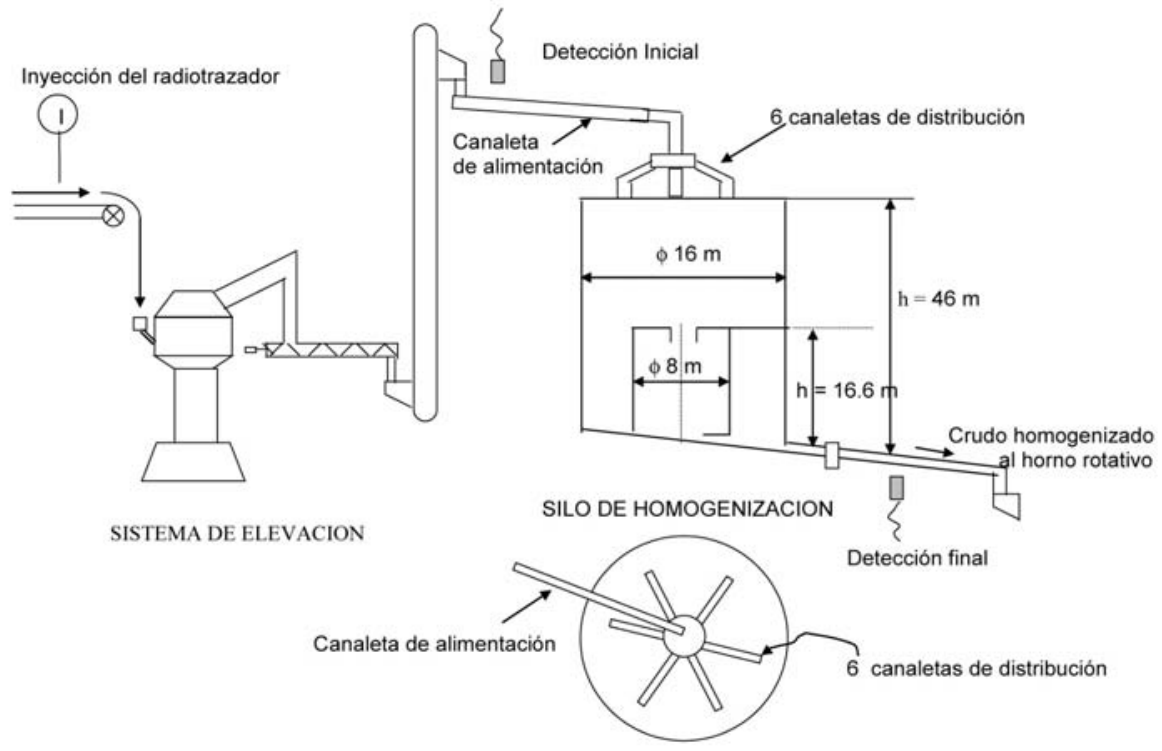

Fig. 3 Disposición de la instalación y arreglo experimental, mostrando los puntos de inyección y detección del radiotrazador

\section{Resultados obtenidos y Cálculos}

La Figura 4 muestra las curvas de distribución obtenidas en tasa de contaje en función del tiempo, para cada una de las salidas de materias del silo de homogenización, Se programó el sistema de medición de tal forma que se realicen contajes de radiación en modo normal a intervalos de 15 minutos en el caso del detector ubicado en la Salida 1 y de 20 minutos para el detector ubicado en la Salida 2. 

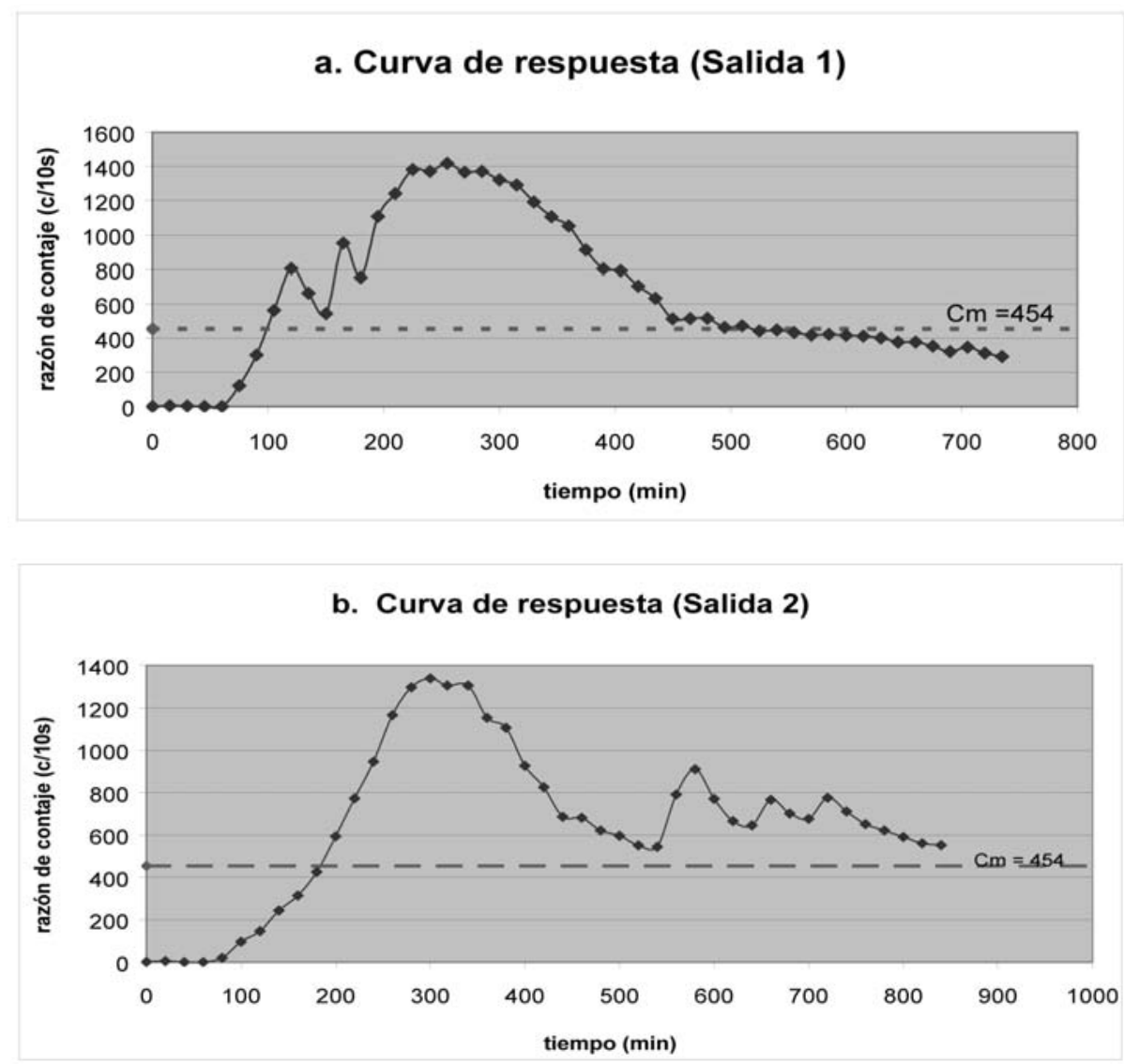

Fig.4 Curvas de respuesta del radiotrazador a la salida del silo de homogenización continua

En las curvas de respuesta $6 \mathrm{a}$ y $6 \mathrm{~b}$ se indica el contaje medio en el caso de que el radiotrazador se hubiese mezclado uniformemente con toda la masa de crudo fino homogenizado que sale del silo durante todo el tiempo total de medición. Estos contajes medios corresponden a 454 cuentas $/ 10$ seg, para el caso de ambos detectores ubicados en las Salidas 1 y 2, respectivamente.

Aplicando la Ecuación 3, con los valores discretos de ambas curvas de respuesta de las Tablas 1 y 2 obtenemos:

$$
\text { Para la Salida } 1 \mathrm{E}=300 \mathrm{~min} .(5,00 \text { h.) } \quad \text { Para la Salida } 2 \quad \mathrm{E}=338 \mathrm{~min} .(5,64 \text { h. })
$$

Observación.- Se ha aplicado la Ecuación 3 considerando la porción principal de las curvas de distribución con sus correspondientes ajustes. Los picos observados antes de la salida de la fracción principal (Curva 6a)y después de la salida de la fracción principal (Curva 6b), serán materia del análisis que sigue a continuación. 


\section{Evaluación de resultados}

- La curva de respuesta para la Salida 1 revela la presencia de trazador por primera vez luego después de 1 hora de iniciadas las mediciones (o una hora después de la inyección del trazador por el techo del silo), momento en que se observa un gradual incremento del contaje de radiación.

- La curva de respuesta para la Salida 1 presenta dos máximos previos (antes de la salida de la fracción principal), cuyas amplitudes son del orden de 90 y 30 minutos en la escala de tiempo. Cerca del $75 \%$ del material marcado deja la Salida 1 en un período de 9 horas, correspondiendo el 25\% restante al volumen muerto del silo (zona de declinación estacionaria de la concentración radiactiva, correspondiendo el primer valor de esta zona al punto de intersección de la curva con la línea de isoconcentración esperada si el mezclado fuera completamente uniforme).

- La curva de distribución para la Salida 2 revela la presencia de trazador por primera vez, luego después de 1 hora de iniciadas las mediciones, en forma similar a la curva de respuesta de la Salida1. A partir de ese momento se observa un incremento gradual del contaje de radiación.

- La curva de respuesta para la Salida 2 presenta una distribución homogénea en las primeras 9 horas, correspondiendo en ese momento a la salida del 52\% del material marcado. A partir de ese instante se observan algunos picos secundarios que corresponden al $28 \%$ del material marcado, hasta completar las 14 horas. El 20\% restante corresponde al volumen muerto del silo (zona de declinación estacionaria de la concentración radiactiva, correspondiendo el primer valor de esta zona al punto de intersección de la curva con la línea de isoconcentración esperada, si el mezclado fuera completamente uniforme). Debe observarse que si se consideran los picos secundarios como parte de la curva principal de distribución, se tendría un acumulado de $80 \%$; valor muy próximo al acumulado de la fracción principal de la Salida 1,

- Los picos previos observados en la curva de respuesta de la Salida 1 no corresponden a corto circuitos del sistema ya que están muy próximos a la fracción principal de la curva. En realidad dichos picos se explican como resultado de las canalizaciones existentes en el interior del silo, debido a la formación de embudos en las zonas próximas a las aberturas por donde el material es conducido hacia la cámara interna; tal como se representa en la Fig, 1. El primer máximo corresponde a la primera abertura localizada en la parte superior de la cámara interna y el segundo máximo corresponde a la segunda abertura localizada en la parte lateral inferior de la cámara interna del silo.

- La curva de respuesta de la Salida 2 no presenta picos previos por lo que se deduce que el efecto de las canalizaciones internas es minimizado con los cambios en los cuadrantes de aireación. Por el contrario aparecen algunas oscilaciones luego de la salida de la fracción principal que corresponden a pequeñas fracciones recirculantes 
de radiotrazador que, saliendo de la cámara interna fluidizada, no logran depositarse totalmente en el depósito adyacente a cada cuadrante, desde donde se retira material homogenizado del silo.

- El volumen muerto teórico estimado con aplicación de la Ecuación 5, sería del orden del $35 \%$, mientras que el valor experimental medio es del $23 \%$, La diferencia la explicamos por que no se conocen valores exactos del grado de compactación del material en las condiciones normales de almacenamiento del silo; habiéndose realizado estimados de volumen y capacidad considerando los valores de densidad sin compactar y compactada indicados anteriormente.

- Cálculo de la Desviación Estándar ( $\sigma$ ) a la salida del crudo homogenizado,- La Tabla 3 muestra los valores de contaje para intervalos de contaje de 1 hora, a partir de los cuales se determinan la desviación estándar de las concentraciones de radiación, a la salida del silo, utilizando la Ecuación 6. La homogenización de la mezcla es caracterizada por la desviación estándar relativa de la concentración radiactiva, conforme se muestra en la misma tabla, para diferentes intervalos de tiempo.

TABLA 2. CÁLCULO DE LAS DESVIACIÓN ESTÁNDAR*

\begin{tabular}{|c|c|c|c|c|c|c|c|c|}
\hline \multirow[t]{2}{*}{$\begin{array}{c}\mathrm{t} \\
(\mathrm{hr})\end{array}$} & \multicolumn{4}{|c|}{$\begin{array}{c}\text { Salida } 1 \\
n=10 \\
C_{m}=454 \text { cuentas } / 10 \text { seg }\end{array}$} & \multicolumn{4}{|c|}{$\begin{array}{c}\text { Salida } 2 \\
n=14 \\
C_{m}=454 \text { cuentas } / 10 \mathrm{seg}\end{array}$} \\
\hline & $\mathrm{c} / 10 \mathrm{seg}$ & $S$ & $\mathrm{~S}_{\mathrm{rel}}(\%)$ & $f$ & c/10 seg & $s$ & $\mathrm{~S}_{\mathrm{rel}}(\%)$ & $f$ \\
\hline 1 & 0 & --- & -- & -- & 0 & -- & --- & --- \\
\hline 2 & 805 & 569 & 25,38 & 1 & 146 & 103 & 77,26 & 1 \\
\hline 3 & 753 & 451 & 0,77 & 0,03 & 426 & 216 & 52,32 & 0,68 \\
\hline 4 & 1373 & 563 & 24,11 & 0,95 & 946 & 417 & 8,15 & 0,11 \\
\hline 5 & 1323 & 555 & 22,20 & 0,87 & 1339 & 561 & 23,53 & 0,30 \\
\hline 6 & 1053 & 503 & 10,80 & 0,43 & 1153 & 555 & 22,24 & 0,29 \\
\hline 7 & 703 & 464 & 2,27 & 0,09 & 826 & 510 & 12,36 & 0,16 \\
\hline 8 & 517 & 447 & 1,65 & 0,07 & 621 & 473 & 4,17 & 0,05 \\
\hline 9 & 448 & 435 & 4,12 & 0,16 & 544 & 445 & 2,03 & 0,03 \\
\hline 10 & 417 & 426 & 6,23 & 0,25 & 771 & 421 & 7,35 & 0,10 \\
\hline 11 & - & - & - & - & 766 & 400 & 11,91 & 0,15 \\
\hline 12 & - & - & - & - & 776 & 382 & 15,81 & 0,20 \\
\hline 13 & - & - & - & - & 621 & 366 & 19,27 & 0,25 \\
\hline 14 & - & - & - & - & 552 & 354 & 22,03 & 0,29 \\
\hline
\end{tabular}

* Se toma como referencia para el cálculo de la desviación estándar, el instante más próximo a la aparición del trazador por cada una de las salidas (dos horas, en este caso, en que se han agrupado los datos a intervalos de una hora).

- Del análisis del cuadro anterior se deduce que para ambas salidas de crudo fino homogenizado, la menor desviación estándar relativa con respecto al valor medio esperado para un mezclado uniforme, es de 8 horas para el caso de la Salida 1 y 9 horas para el caso de la Salida 2, sin considerar los fenómenos que dan lugar a los picos previos a la fracción principal de la curva DTR en el caso de la Salida 1, y a los picos 
posteriores a la fracción principal de la curva DTR en el caso de la Salida 2; aspectos que ya fueron analizados. Dichos fenómenos originan algunos valores bajos de $\sigma_{\text {rel }}$ (\%), como por ejm, 0,77 (Salida 1) y 8,15 (Salida2), los cuales no deben considerarse para efectos del estimado del grado de homogenización.

- Tomando como base el tiempo medio de residencia determinado experimentalmente para ambas salidas, se establece un factor de reducción de la desviación estándar de 0,87 para la Salida 1 y de 0,30 para la Salida 2. Estos valores son representativos del Grado de Homogenización, resultando bastante bueno para el caso de la Salida 1 y al parecer algo bajo para el caso de la Salida 2 .Esto último se puede explicar por tres razones: a) No hay suficiente precisión para el estimado del factor para la Salida 2 porque la DTR es muy alejada de una distribución normal por la presencia de picos secundarios de recirculación de material, b) Se puede obtener unas cantidad mayor de valores de desviación estándar considerando la totalidad de los puntos de la Tabla 1 , en vez de 14 valores a intervalos de una hora, incluyendo los picos secundarios a la distribución principal, y c) La fracción principal de la curva de distribución de la Salida 2 (52\%), es mucho menor que la de la Salida1(75\%), debido a los fenómenos ya mencionados.

- Por las características de las curvas de respuesta obtenidas, son más confiables los resultados obtenidos en la Salida 1. Sin embargo el tiempo de residencia medio es muy similar en ambas descargas de crudo fino homogenizado.

- El volumen efectivo del silo es del órden del 77\% (valor medio que es muy próximo en ambas descargas).

\section{Conclusiones y recomendaciones}

- El silo de homogenización continua investigado funciona eficientemente en lo que respecta a la obtención de un crudo fino de composición química uniforme, a pesar de tener un volumen inactivo considerable.

- Lo anterior se confirmó con el análisis realizado al crudo fino homogenizado que presenta una composición química similar a la teórica, calculada teniendo en cuenta la abundancia relativa con que ingresan las materias primas del cemento y la composición química de las mismas.

- Durante la descarga del silo, los estratos son distorsionados debido a la formación de embudos descendientes de material, que luego son entremezclados; asegurando la participación de diferentes grupos de secciones de material de diferente composición química, en el proceso de mezcla y homogenización. De esta manera se mezclan continuamente la mayor cantidad posible de estratos de material, provocando un buen efecto de homogenización. 
- A fin de minimizar el efecto de las canalizaciones en el interior del silo, es recomendable considerar la utilización de una cámara interna cónica o la misma cámara cilíndrica pero con techo cónico, con el vértice hacia arriba y con una abertura de succión en la punta del cono y otra abertura de succión en la parte inferior. Esto además ayudaría a disminuir considerablemente los volúmenes muertos por el evidente desplazamiento del material cercano a la pared del silo; el mismo que encontraría una inclinación favorable para su movimiento hacia las succiones.

- Además de la medida anteriormente citada, se podrían minimizar las canalizaciones con un incremento del flujo de aire introducido en los cuadrantes, o aumentando el tiempo de aireación manteniendo el mismo flujo de aire. En las condiciones de la experiencia el flujo de aire era de $1 \mathrm{Nm}^{3} / \mathrm{m}^{2} \cdot \mathrm{min}$.

\section{Referencias bibliográficas}

SCHARLTON J. / HESLOPARD I.A. / JHONSON P. Radioisotope technique for the investigation of process, Imperial Chemical Industries Billingham/Cleveland - England.

Worthington, R.; L, PETERS C. «Revamping inefficient raw meal blending silos».- Ciments, Bétons, Platres, Chaux, № 775-6/8.

BARANYAI, L. «Radiotracerinvestigations ofcementrawmeal homogenizers». Zement-Kal-Gips-№.4/1982

KRAUB, VON W. «Storage and blending silo technology with tunnel discharge system and new types of blending silos»,- Zement-Kal-Gips-№.12/1976.

THYN, J. «Determination of the sequence of discharge of particulate solids from bunkers and silos by tracer techniques».- Chem. E. Symposium Series № 65.

SEBASTIÁN, C. «Evaluación y Optimización de Sistemas de Molienda de Clinker de cemento utilizando Trazadores Radiactivos».- Informe Técnico. Lima - Perú, 1995.

SEBASTIÁN, C.; MAGUIÑA, J.; VÁsQUEZ, J. «Cuantificación de pérdidas de material sólido en plantas de procesamiento de arcillas».- Informe Técnico, Lima-Perú.

VELA, R. «Análisis de la hidrodinámica de sistemas de flujo continuo utilizando trazadores radiactivos».- Informe Técnico, Lima - Perú, 1996.

THERESKA, J. «Radiotracer Methodology and Technology».- IAEA, NAPC, Industrial Applications and Chemistry Section.- Vienna, February 1999.

IAEA «Emerging New Applications of Radiotracers in Industry».- Vienna, Austria. 1997.

IAEA «Practical Guidebook for Radioisotope-Based Technology in Industry».- IAEA/RCA-RAS/8/078, 1999.

IAEA «Radiotracer Technology for Engineering Unit Operation Studies and Unit Processes Optimization».- Technical Report, Krakow - Poland, 1999. 